\title{
Articulated blade tip devices for load alleviation on wind turbines
}

\author{
Carlo L. Bottasso ${ }^{1,2}$, Alessandro Croce ${ }^{1}$, Federico Gualdoni ${ }^{1}$, Pierluigi Montinari ${ }^{1}$, and \\ Carlo E. D. Riboldi ${ }^{1}$ \\ ${ }^{1}$ Wind Energy Institute, Technische Universität München, 85748 Garching b. München, Germany \\ ${ }^{2}$ Dipartimento di Scienze e Tecnologie Aerospaziali, Politecnico di Milano, 20156 Milan, Italy \\ Correspondence to: Carlo L. Bottasso (carlo.bottasso@ tum.de)
}

Received: 25 May 2016 - Published in Wind Energ. Sci. Discuss.: 2 June 2016

Accepted: 19 October 2016 - Published: 1 December 2016

\begin{abstract}
This paper investigates the load alleviation capabilities of an articulated tip device, where the outermost portion of the blade can rotate with respect to the rest of the blade. Passive, semi-passive and active solutions are developed for the tip rotation. In the passive and semi-passive configurations tip pitching is mainly driven by aerodynamic loads, while for the active case the rotation is obtained with an actuator commanded by a feedback control law. Each configuration is analyzed and tested using a high-fidelity aeroservoelastic simulation environment, by considering standard operative conditions as well as fault situations. The potential benefits of the proposed blade tip concepts are discussed in terms of performance and robustness.
\end{abstract}

\section{Introduction and motivation}

The cost of energy $(\mathrm{CoE})$ is the key parameter that determines the success of an energy source. In recent years, both industry and the wind energy scientific community have focused their efforts on the reduction in the $\mathrm{CoE}$, with the goal of increasing the competitiveness of energy from wind with respect to other technologies. A reduction in the $\mathrm{CoE}$ can be obtained by a variety of means, one of the most significant effects coming from an increase in the annual energy production (AEP). AEP can be increased by improving the aerodynamic efficiency of the rotor and by harvesting a greater amount of energy with larger swept areas and taller towers. Because of this, together with other scale benefits typically associated with larger wind turbines, there is a very clearly marked trend towards bigger machines. In the offshore case, where logistics and transportation are very different from onshore, the tendency towards very large wind turbines is even clearer, the optimum size not having been reached yet.

To satisfy this growth trend, the simple upscaling of existing machines is unfeasible. In fact, as cost is typically well correlated with mass and mass with volume, a naive scaling would translate into an unacceptable cubic growth of cost. Among other approaches, load alleviation techniques help address this issue, increasing the efficiency of the aerostructural configuration and limiting the cost growth rate of wind turbine components (Thresher et al., 2008).

The mitigation of loads can be obtained by fullspan/distributed and passivelactive solutions. Full-span solutions involve the response of the entire blade. Individual pitch control (IPC) is a full-span active technique, which is seeing an ever increasing acceptance by industry, while bendtwist coupling (BTC) is an example of the full-span passive category (Bottasso et al., 2013a). Although often very effective, any full-span solution is inherently somewhat limited in bandwidth, due to the inertia and nonlocal response of the blade. Moreover, pitch-bearing fatigue and the usage of the pitch actuators limit the application of full-span active techniques. Distributed solutions, on the other hand, locally affect the flow using flaps, tabs or other devices. The local nature of these solutions allows in principle for a higher bandwidth both in space and in time, which could potentially result in an even higher reduction in loads. This should however be traded with their higher complexity, which might in turn affect $\mathrm{CoE}$ because of higher production and maintenance costs and/or decreased availability. 
Numerous distributed active solutions for horizontal axis wind turbines (HAWTs) have been explored, often inspired by aeronautical applications. At present, the most mature applications appear to be the ones based on trailing edge flaps (Andersen et al., 2010; Lackner and van Kuik, 2010; Barlas et al., 2011; Bergami and Poulsen, 2015), although alternative solutions based on micro-tabs and compliant structures have also been considered (Chow and van Dam, 2007; Lachenal et al., 2013).

Passive distributed techniques were first developed for aeronautical applications. An early example of passive load alleviation is reported by Donely and Shufflebarger (1940), in which a long-period overbalanced flap was used to reduce airplane accelerations due to atmospheric gusts. A comparison of different passive devices for the alleviation of vibratory loads on helicopter rotors is described by Bielawa (1984). This study identified the passive blade tip concept as the most promising technique to improve the aeromechanical qualities of the rotor. Blades were modified in their outermost portion to install a free pitching tip. The relative rotation between tip and the inner blade was driven by the aeroelastic loads, and the device parameters were tuned to achieve the desired dynamic response.

The design of the passive tip is the outcome of intense research activity at NASA in the 80s. The simulation of a passive tip concept is described by Stroub (1982), aiming at a more uniform airload distribution during the blade revolution by self-adjusting blade tips. Analytical results showed an improvement in lift generated by the rotor in cruise conditions and a reduction in drag and the power required. Since the mean relative rotation of the tip is related to the restraining moment at the hinge, a preload was used as a tuning parameter to modulate the blade tip angle of attack and the resulting aerodynamic forces. The passive tip concept was also validated through experiments (Stroub, 1985), which confirmed a considerable reduction in required power in high thrust conditions. This result is related to a favorable influence of the blade tip negative pitch angle with respect to the inboard blade portion. Furthermore, the flapwise and control loads were reduced considerably, although no positive effects were observed on the lead-lag loads. Additional studies focused on the configuration of a passive torque controller used to adjust the preload (Young, 1986). This fully passive mechanism converts centrifugal loads in a preset torque at the movable tip. Including considerations on simplicity and reliability, the most promising solution appeared to be one that generates the output torque from the tensile loading of two twisted wire straps (Louie, 1988).

Notwithstanding these promising results, passive tips have not been adopted by the helicopter industry. Although active flaps have also not yet arrived on the market, they have seen some significant demonstration by industry (Konstanzer et al., 2008). In fact, in aeronautical applications higher levels of complexity are acceptable if they entail superior performance, weight savings or the improvement of relevant met- rics. Therefore, in this case active flaps might be in general more interesting than passive ones. The situation is different in the wind energy case, where the main (often unique) driver is the CoE. In this case, availability and maintenance costs are of paramount importance. From this point of view, deploying a wind turbine with active flaps in the field still seems to be a very significant challenge. Therefore, for wind energy applications a passive solution might be more appealing than an active one if the former implies greater simplicity, robustness and ease of repair than the latter.

Among the first applications of passive distributed solutions in wind energy is the airfoil camber regulation described by Lambie et al. (2011). This passive device adapts blade camber to alleviate pressure fluctuations. The desired behavior is obtained by tuning the structural properties of the device, consisting of a spring and damper. A preliminary validation was performed on a typical section model, while a more recent analysis is reported in Marten et al. (2015), where a nonlinear lifting-line free-vortex wake model is employed to assess the performance of the passive device on a multi-MW HAWT. Results indicate a reduction in the standard deviation of blade root bending moments, although a single simulation was considered.

Another passive camber solution is based on bistable composite structures (Arrieta et al., 2014). In this case, the airfoil camber variation is triggered by the aerodynamic loads that modify the equilibrium condition of a compliant structure with embedded multi-stable elements. This technique results in a discrete control action because only a finite number of stable configurations are possible. Furthermore, an external load has to be provided to restore the original blade camber.

A fully articulated passive flap was first proposed by Bottasso et al. (2015b). The idea is in this case to offset the flap center of gravity forward of the hinge line. This way, flapwise accelerations of the blade excite a response of the flap that, by changing the airfoil camber, tends to oppose the acceleration itself, thereby attenuating blade loading and in turn fatigue. The flap is also aerodynamically balanced, in the sense that it is designed not to respond to the deliberate changes in the angle of attack imposed by the wind turbine control system. Multiple load cases were considered through a loose coupling procedure based on a state-of-the-art aeroservoelastic simulator and a typical section model, indicating very promising performance.

As the literature shows, a few recent studies have considered passive flaps for HAWTs. However, one of the most promising solutions for rotorcraft applications, the blade free tip, was only explored and tested during the 90s by the FLEXHAT program (van Kuik and Dekker, 1992; Hagg et al., 1993). That study considered a two-bladed HAWT equipped with an elastomeric teeter, a flexbeam allowing a limited flapping motion, as well as two passively activated blade tips. Despite the considerable advantages in terms of load reduction, the solution was not developed further due to its inherent complexity. 


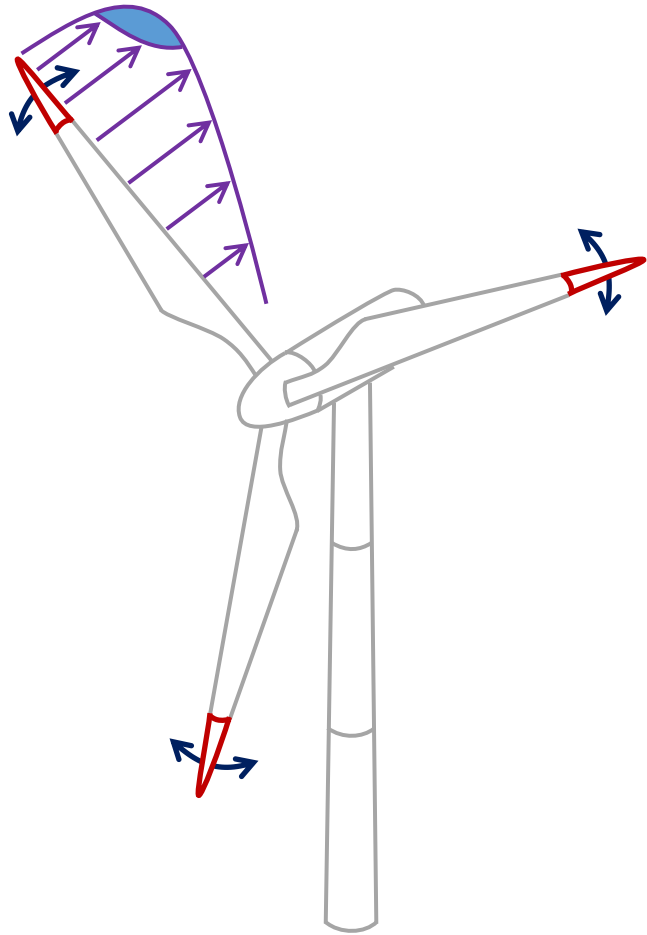

Figure 1. Articulated blade tip concept for load alleviation.

The present study tries to further explore the tip concept, investigating various solutions for the alleviation of loads on multi-MW HAWTs (see Fig. 1). Passive, semi-passive and active solutions are considered in order to provide a general overview of the possible range of configurations and their respective performance. The passive solution is purely activated by aerodynamic loads, while the semi-passive one uses an active component to apply a varying restraining torque to limit mean tip deflections according to the machine operating condition. Finally, the active solution uses an actuator to drive the tip deflection based on a feedback control law. Each configuration is analyzed in detail, including the tuning of the respective parameters. Performance is assessed using the accepted international certification standards within a highfidelity aeroservoelastic simulation environment.

The paper is organized as follows. Section 2 considers the tip design problem. Passive and semi-passive configurations are examined first, providing some general guidelines and a preliminary sizing of the main system parameters for the aeroelastic integration of the devices on board the wind turbine. The active solution is then introduced, and its control algorithm is tuned. Next, Sect. 3 compares fatigue and ultimate loads as well as off-design conditions. Finally, conclusions and an outlook on possible future developments are reported in Sect. 4.

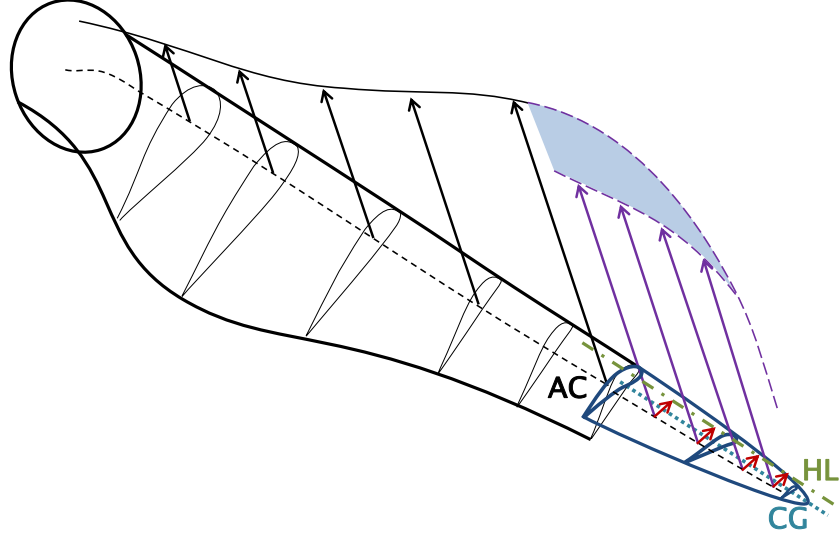

Figure 2. Wind turbine blade with articulated tip.

\section{Design of blade tip devices}

The design of the blade tip focuses here on the properties of the hinge connecting it to the rest of the blade, while the external blade shape is kept constant. This simplification distinguishes the effects of the tip motion per se from further possible effects that could be obtained by modifying its aerodynamic shape. While the approach might be suboptimal, a specific tailoring of the aerodynamic characteristics of the tip can be analyzed at a later stage.

\subsection{Passive and semi-passive configurations}

The device design aims at optimizing the tip motion in order to mitigate loads. The positions of the hinge line (HL), of the tip aerodynamic center (AC) and of the center of gravity (CG) (see Fig. 2) play a crucial role in determining the physical phenomena contributing to load alleviation.

If the hinge line is close to the aerodynamic center of the blade tip, then the aerodynamic moment is nearly independent of angle of attack changes. Therefore, the device behavior is mainly driven by the inertial response of the blade tip if its center of gravity is offset with respect to the hinge. This is the same load alleviating mechanism used by Bottasso et al. (2015b) for their passive trailing edge flap. By contrast, if the hinge line is away from the blade tip aerodynamic center while the center of gravity is not, then the response is mainly driven by aerodynamic loads. In particular, when the hinge line is forward of the aerodynamic center, an increase in the angle of attack at the blade tip will induce an increase in lift and, consequently, a nose down moment at the hinge that will induce a pitch down rotation. This will eventually oppose the original increase in the angle of attack, thereby realizing a load-mitigating action.

Both the inertial and aerodynamically driven solutions can be used for designing passive load-mitigating devices. However, while the former proved to be very effective for the flap case (Bottasso et al., 2015b), the latter seems to be better 
suited to the tip case considered in the present study. Several factors make the inertial-driven solution difficult to implement for a tip device. First, a flap is characterized by the hinge moment rate of change with respect to both angle of attack and flap deflection changes, two parameters that can to a large degree be set independently of each other. By contrast, a tip device is only characterized by its sole hinge moment rate of change with respect to the angle of attack; in addition, the moment with respect to the aerodynamic center is not null because of the non-null camber of the tip airfoils. Therefore, it is much harder for the tip case to obtain a good alleviating performance and small sensitivity to disturbances such as gravity and centrifugal loading. In addition, a significant mass ballast is needed to obtain the necessary inertial effects, ballast that in turn lowers the blade natural frequencies and may negatively affect loading. Based on these considerations, the aerodynamically driven solution is adopted for the present study.

The hinge location is a compromise between the weathercock tendency of the blade tip, which suggests a forward position, and a desire to limit inertial couplings, which suggests a hinge position close to the center of gravity of the tip.

The spanwise extent of the blade tip was optimized with the help of a parametric analysis, considering a trade-off among blade root load alleviation, loading at the hinge and impact on power capture.

The wind turbine is operated with a variable-speed pitchtorque control strategy, including the partial load regime (or region II) from cut-in to rated speed and the full-load regime (or region III) from rated to cut-out speed (Bottasso et al., 2011). The best possible aerodynamic performance is sought in region II to optimize power capture. Therefore, the mean misalignment of the tip with respect to the rest of the blade should be as small as possible not to negatively affect the rotor efficiency. On the other hand, an excess of power is available in region III, so that a mean misalignment of the tip is permissible in this case as it would be readily compensated for by the control system without incurring any AEP loss.

A torsional spring and torque preload are used at the hinge with the aim of controlling the tip response. The tip pitch dynamic equilibrium is

$J_{\theta} \ddot{\theta}+K_{\theta} \theta=M_{\mathrm{p}}+M_{\mathrm{a}}$,

where $\theta$ is the tip pitch rotation, $J_{\theta}$ the tip inertia, $K_{\theta}$ the torsional spring stiffness at the hinge, $M_{\mathrm{p}}$ the hinge preload and $M_{\mathrm{a}}$ the aerodynamic moment. The primary device design parameters are $K_{\theta}$ and $M_{\mathrm{p}}$.

The torsional spring $K_{\theta}$ was calibrated to limit the tip pitch oscillation amplitude. This tuning was performed by running aeroservoelastic simulations in steady and turbulent conditions for varying wind speeds spanning the entire operating range of the machine and identifying an optimal compromise between fatigue alleviation and power loss. Although in principle the spring stiffness might be scheduled with respect to
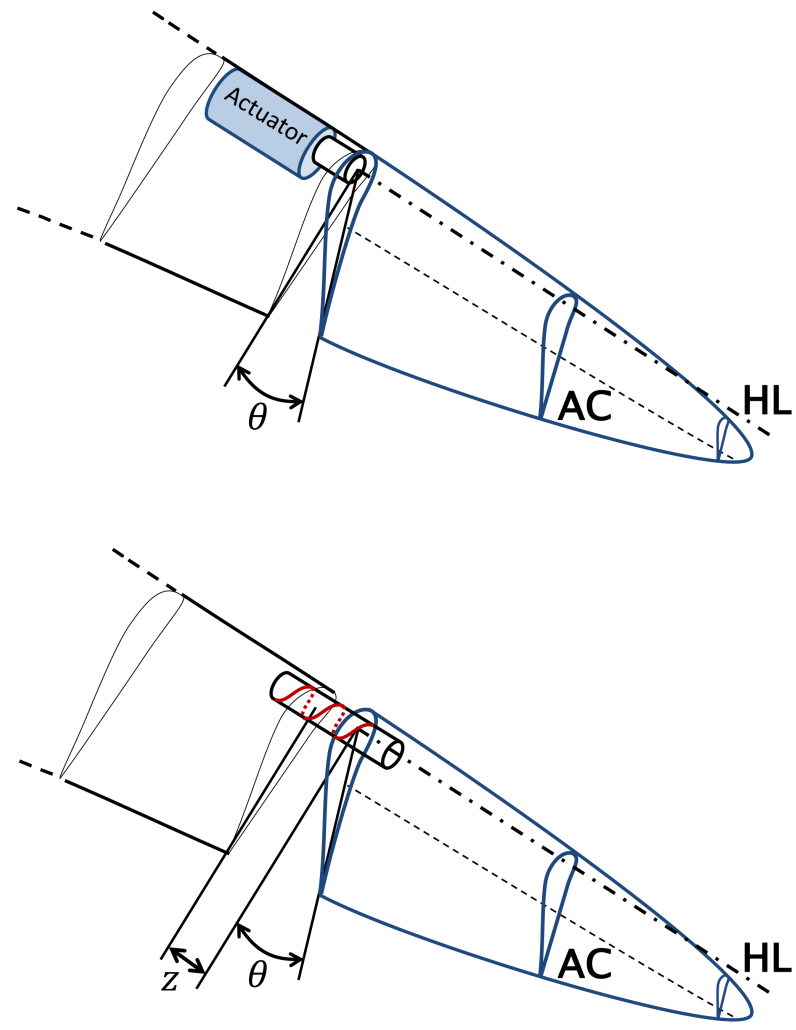

Figure 3. Top: semi-passive tip configuration. Bottom: passive tip configuration.

the operating condition, it was found that a constant average value was a simpler and similarly effective solution.

The tip mean misalignment is controlled by providing a torque preload $M_{\mathrm{p}}$ at the hinge. As the aerodynamic loading at the tip, and hence its mean moment at the hinge, depends on the operating condition, the preload should be varied on account of the operating point at which the machine is functioning. In the semi-passive configuration the preload is generated by an actuator, while in the passive case by a mechanical device that produces a torque in response to the centrifugal loads generated by the blade rotation. In both cases, the resulting preload at the hinge is directly related to the rotor angular speed.

A sketch of the passive and semi-passive configurations is found in Fig. 3.

As the preload is related to the operating point, its value can be computed in steady-state normal wind profile (NWP) conditions using a complete aeroservoelastic model of the wind turbine, scanning wind speeds from cut-in to cut-out. To speed up the identification of the necessary preload value, at each wind speed a simulation was run where the relative rotation in the tip hinge was set to zero. Once the solution had settled into a periodic cycle, the mean value of the resulting torque in the hinge was used as the preload value for that operating condition. 
In principle, the preload could be scheduled with respect to the mean wind speed or to the rotor angular velocity. The former option is more complicated and possibly less reliable because it requires an observer to estimate the rotorequivalent wind speed. By contrast, scheduling the preload with respect to the rotor angular velocity is simpler, since measurements of the rotor speed are available on board wind turbines. As the angular velocity is constant in region III, a constant preload above rated wind speed will result in a nonnull mean misalignment of the tip. This is not a problem, as there is a power excess in this condition, so that a less efficient rotor does not pose any concern. The situation would be different for a machine with a transition region II1/2 in between regions II and III - which happens whenever the rotor speed hits its upper limit before rated power is reached - where scheduling with respect to rotor speed alone might incur power losses.

For the semi-passive configuration, an actuator applies the necessary preload torque at the hinge based on a look-up table storing the load-rotor speed map $M_{\mathrm{p}}=M_{\mathrm{p}}(\Omega)$ obtained in the previously described analyses, where $\Omega$ is the rotor angular velocity. No feedback regulation is involved, and the actuator simply uses the filtered (to remove fast fluctuations and noise) rotor speed as feedforward information.

The passive configuration uses centrifugal forces caused by the rotor angular rotation to generate the necessary preload $M_{\mathrm{p}}$, without using active components. To this end, in this paper we consider the mechanical device described in Stroub (1982), characterized by a screw joint that relates any linear displacement $z$ of the tip parallel to its hinge axis to a corresponding rotation $\theta$ about the same axis, i.e., $z=\tau \theta$, where $\tau$ is the screw joint helical pitch or transmission ratio. The actual mechanical design of this device is beyond the scope of this study, and its characterization is here limited to the evaluation of its parameter $\tau$. The passive tip pitch dynamic equilibrium can be written as

$J_{\mathrm{PT}} \ddot{\theta}+K_{\mathrm{PT}} \theta=\tau\left(F_{\mathrm{c}}+F_{\mathrm{g}}\right)+M_{\mathrm{a}}$,

with

$$
\begin{aligned}
J_{\mathrm{PT}} & =J_{\theta}+\tau^{2} m, \\
K_{\mathrm{PT}} & =K_{\mathrm{PT} \theta}+\tau^{2} K_{z},
\end{aligned}
$$

where $J_{\mathrm{PT}}$ and $K_{\mathrm{PT}}$ are the total inertia and torsional stiffness of the passive tip device. These include the proper inertia of the tip $J_{\theta}$ and the hinge spring $K_{\mathrm{PT} \theta}$, in addition to terms contributed by the screw joint, $m$ being the tip mass and $K_{z}$ the screw linear displacement stiffness. In Eq. (2), $F_{\mathrm{c}}$ and $F_{\mathrm{g}}$ are the centrifugal and gravitational forces, respectively, projected onto the blade spanwise direction. To a first approximation, the effects due to blade out-of-plane and inplane motion, as well as the contributions of rotor cone and uptilt angles, are neglected. Therefore, the centrifugal force is expressed as

$F_{\mathrm{c}}=m(r+z) \Omega^{2}$, where $r$ is the radial position of the tip center of gravity. Inserting Eq. (4) into Eq. (2), one gets

$J_{\mathrm{PT}} \ddot{\theta}+\left(K_{\mathrm{PT}}-m \tau^{2} \Omega^{2}\right) \theta=\tau m r \Omega^{2}+\tau F_{\mathrm{g}}+M_{\mathrm{a}}$.

The gravitational force is

$F_{\mathrm{g}}=m g \cos \psi$,

where $\psi$ is the blade azimuthal position and $g$ the acceleration of gravity. Since $F_{\mathrm{g}}$ is a periodic disturbance with zero mean over a revolution, the transmission ratio $\tau$ is chosen such that the first term on the right-hand side of the equation balances the aerodynamic moment at the hinge line, leading to

$\tau=-\frac{M_{\mathrm{a}}}{m r \Omega^{2}}$.

An average value of $M_{\mathrm{a}}$ over the most likely operating conditions (between 7 and $9 \mathrm{~m} \mathrm{~s}^{-1}$, according to the Weibull distribution considered here) is used to compute $\tau$.

The value of the hinge spring stiffness for the passive tip case was set by requiring this device to have the same modal frequency as the semi-passive case, which is readily computed from Eq. (1) as $\omega_{\mathrm{SP}}^{2}=K_{\theta} / J_{\theta}$. By setting $K_{z}=0$ and using Eq. (5), one gets

$K_{\mathrm{PT}, \theta}=\omega_{\mathrm{SP}}^{2} J_{\mathrm{PT}}+m \tau^{2} \Omega^{2} \approx \omega_{\mathrm{SP}}^{2} J_{\mathrm{PT}}$,

where the term depending on angular velocity was dropped because it was negligible. This choice results in a hinge stiffness that, conveniently, does not depend on the operating condition, as in the semi-passive case.

It should be stressed that this is not the only possible criterion to determine the hinge spring stiffness for the passive tip case. In fact, the tip mode could in principle be placed anywhere in the spectrum, as long as it does not create resonant conditions with the per-rev harmonic excitations and with other natural frequencies of the machine. On the other hand, the present approach seemed to work well in practice. In fact, raising this frequency by increasing the spring stiffness limits the tip pitch oscillations, in turn reducing its authority. The opposite approach of lowering the frequency by softening the spring has the effect of increasing the disturbance caused by gravity. In fact, gravity cyclically pulls on the blade tip, creating a radial displacement that, through the screw joint, induces a pitch rotation, which in turn creates a 1P disturbance. The present approach was found to provide a good compromise between these two contrasting requirements, although a further fine-tuning of the parameters is probably still possible. 
Table 1. Principal parameters of the DTU $10 \mathrm{MW}$ RWT.

\begin{tabular}{lr}
\hline Parameter & Value \\
\hline Rated power & $10 \mathrm{MW}$ \\
Wind class & IEC 1A \\
Rotor diameter & $178.3 \mathrm{~m}$ \\
Hub height & $119.0 \mathrm{~m}$ \\
Rated wind speed & $11.4 \mathrm{~m} \mathrm{~s}^{-1}$ \\
\hline
\end{tabular}

\subsection{Reference wind turbine and simulation environment}

The blade tip devices are sized and studied with application to the $10 \mathrm{MW}$ Reference Wind Turbine (RWT), developed by Danmarks Tekniske Universitet (DTU) (Bak et al., 2013). Some of the principal parameters of the machine are reported in Table 1, while the full database can be downloaded at the project website (DTU RWT, 2015).

All simulations are performed with an aeroservoelastic model of the wind turbine implemented with the flexible multibody program Cp-Lambda (see Bottasso et al., 2006, and references therein). The baseline regulation strategy is provided by an external library implementing the control routines reported in Hansen and Henriksen (2013). Based on a parametric study, the spanwise tip length was set to $15 \%$ of the blade length, while the tip hinge line was located at $19.7 \%$ of the local blade chord from the leading edge. The tip is connected by a revolute joint to the rest of the blade for the semi-passive and active configurations. In both cases, the hinge rotation is driven by an actuator, modeled as a secondorder system. For the passive case, the tip is connected to the blade by a screw joint. In all cases, tip excursions are limited to $\pm 20^{\circ}$ by unilateral contact conditions in the joint.

It was decided to operate all devices both in the partialand full-load regions. Although operation in partial load conditions may affect power output, there is also a significant accumulation of fatigue damage around rated wind speed for sites with low most-probable winds. However, it is also clear that, depending on the characteristics of machine and site, the sole operation in full-load conditions might be preferable.

The aerodynamic model is based on standard blade element momentum (BEM) theory (Hansen, 2008), which is customarily used for the analysis of loads on wind turbines. This approach is not able to model the shed vortex at the blade-tip junction (Stroub et al., 1986). This vortex induces a velocity field that modifies the orientation of the lift vector at the tip, in turn causing a variation of induced drag (Martin and Fortin, 1988). Since in a passive solution aerodynamic loads contribute to the motion of the tip device, this modeling deficiency may also affect the accuracy of the predicted pitch motion. This is one of the main approximations of the present work, which will require further investigation by more sophisticated aerodynamic models.

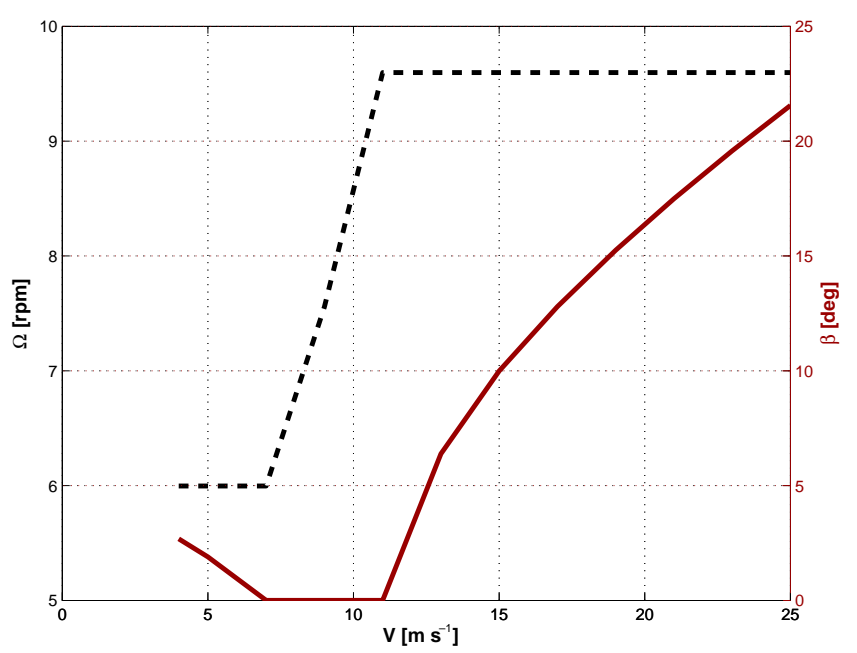

Figure 4. Rotor speed $\Omega$ and blade pitch $\beta$ vs. hub-height wind speed $V$.

\subsection{Sizing of the passive and semi-active solutions}

The wind turbine operating range is first analyzed in NWP conditions (IEC, 2005). The associated rotor speed and blade pitch settings vs. hub-height wind speed are shown in Fig. 4.

Nominal values of the torque preload $M_{\mathrm{p}}$ as a function of wind speed were obtained by constraining to zero the tip rotation at the hinge and measuring the resulting internal moment. The result is shown at the top of Fig. 5, using a dash-dotted line: by prescribing this preload at the hinge, one would obtain a zero mean misalignment of the blade tip. Since the preload is adjusted based on rotor speed both for the passive and the semi-passive configurations, this reference preload can be followed only between $7 \mathrm{~m} \mathrm{~s}^{-1}$ and rated wind speed, when the rotor speed does indeed change (see Fig. 4).

As shown in the figure, for lower and higher wind speeds the actual preload provided by the passive and semi-passive solutions remains constant, implying that the blade tip will have a non-zero mean pitch offset with respect to the blade. The preload can be actively changed by a torque actuator in the semi-passive tip solution, so that the provided preload exactly follows the nominal one in this case. For the passive configuration, the preload is obtained by a constant transmission ratio $\tau$ connecting tip spanwise displacements with tip pitch rotations, which, as shown in the figure, still approximates very well the nominal preload behavior.

Figure 5 on the bottom shows with a dash-dotted line the hinge spring stiffness that would result in a $\pm 10^{\circ}$ oscillation of the tip in NWP conditions. As this stiffness changes little with respect to wind speed, it was approximated with a constant value for the semi-passive case, further tuned with the help of turbulent analyses. From a practical point of view, a constant spring stiffness is useful because it reduces the complexity of the device. As previously explained, the hinge 

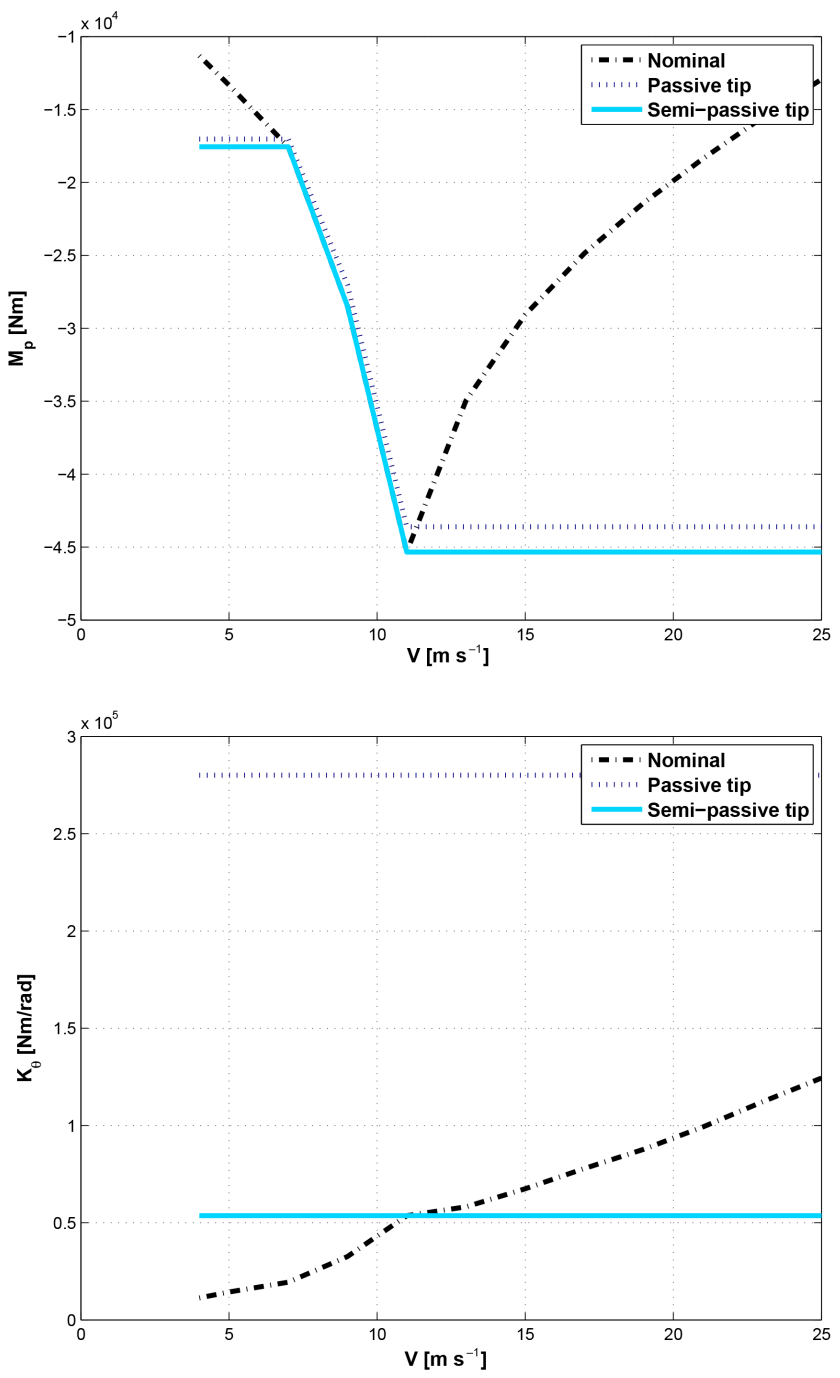

Figure 5. Top: preload $M_{\mathrm{p}}$ at the hinge line vs. hub-height wind speed. Bottom: hinge stiffness $K_{\theta}$ vs. hub-height wind speed.

stiffness for the passive configuration differs from the one of the semi-passive case. In fact, since the transmission ratio of the screw joint increases the torsional inertia of the blade tip, the hinge stiffness was increased to keep the tip mode at the same frequency in both solutions.

Table 2 reports the modal frequencies of the rotating blade in a vacuum at rated speed, for the baseline blade and the semi-passive and passive solutions. Minor differences are due to the adoption of a constant transmission ratio $\tau$, which however is important for the simplicity of the device. The blade tip mode is clearly distinct from the lower blade frequencies, limiting the risk of aeroelastic interactions.

\subsection{Active configuration}

Besides the passive solutions described earlier, tips can also be used for active feedback control. In this case, pitch mo-
Table 2. Modal frequencies of the rotating blade in a vacuum (in $\left.\operatorname{rads}^{-1}\right)$.

\begin{tabular}{lrrr}
\hline Mode & Baseline & Semi-passive tip & Passive tip \\
\hline First flap & 4.08 & 3.99 & 3.99 \\
First edge & 5.67 & 5.40 & 5.39 \\
Second flap & 10.3 & 10.9 & 10.8 \\
Second edge & 15.6 & 16.0 & 15.9 \\
Third flap & 20.0 & 21.9 & 21.9 \\
Tip mode & - & 25.2 & 25.1 \\
Third edge & 31.2 & 33.0 & 32.6 \\
\hline
\end{tabular}

tions are actively driven by tip actuators. Due to the lower inertia of the tip with respect to the entire blade, tip-based active control might have a higher bandwidth than full-span pitch control. In addition, as the tip has a high moment arm with respect to the blade root, even relatively small changes in the aerodynamic loads might have significant repercussions on the overall loading of the rotor. Both of these effects might be especially visible for larger turbines, although a detailed investigation of scale effects is beyond the scope of the present work.

In this paper, cyclic pitch control of the tips is used for the reduction of rotor moments in the fixed system, using a formulation similar to one used for classical full-span IPC (Bossanyi, 2003a, b, 2005; Leithead et al., 2009; Bottasso et al., 2013b). Blade bending moments are measured by load sensors at the blade roots and transformed first into out-ofthe-rotor-plane moments and then into direct $M_{\mathrm{d}}$ and quadrature $M_{\mathrm{q}}$ moments in the fixed frame by the Coleman transformation (Johnson, 2013).

After filtering to remove frequencies at and above 1P, reference loads are subtracted from the Coleman-transformed moments, yielding the delta loads used for feedback $\Delta M=$ $M-M^{*}(\bar{V})$ for both the $\mathrm{q}$ and $\mathrm{r}$ components, where $\bar{V}$ is a slowly varying moving average of the wind speed used for scheduling the reference loads. The use of delta loads is useful because of the lower authority of tip pitch control compared to full-span pitch control. In fact, by cyclically pitching the whole blade, full-span pitch control can very significantly reduce the mean value of fixed-frame loads, which is typically not possible with the sole use of tips.

A proportional-integral (PI) controller is then formulated in the fixed frame, giving

$\beta=k_{\mathrm{P}} \Delta M+k_{\mathrm{I}} \int_{0}^{t} \Delta M \mathrm{~d} t$,

where $k_{\mathrm{P}}$ and $k_{\mathrm{I}}$ are the proportional and integral gains, respectively. The same control law is used for the direct and quadrature components, yielding both the $\beta_{\mathrm{d}}$ and $\beta_{\mathrm{q}}$ control inputs in the fixed frame, which are finally transformed back into the rotating system via Coleman's inverse transform. 


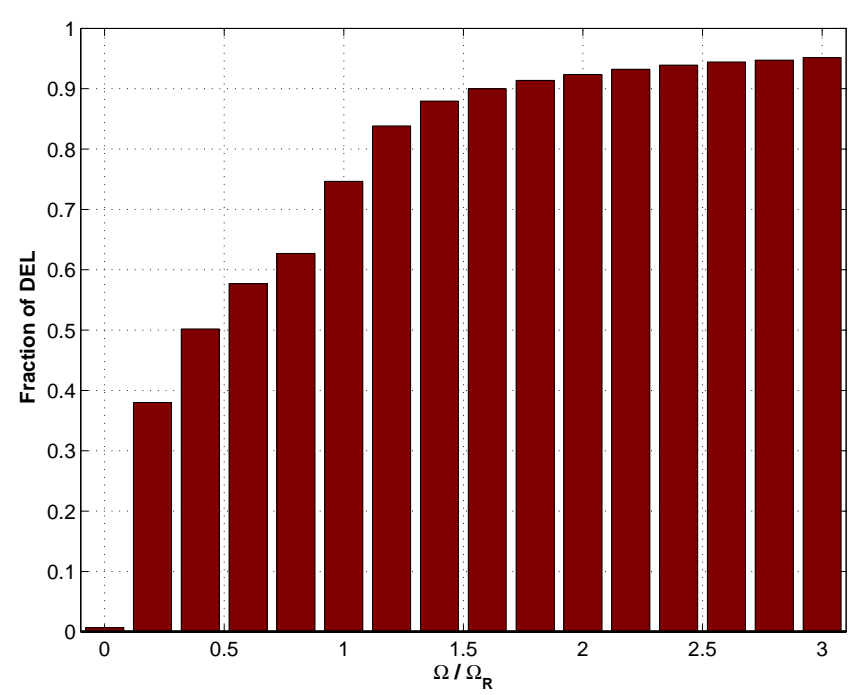

Figure 6. Normalized blade root lifetime DEL bending moment, plotted as a function of load frequency.

This IPC formulation results in a 1P tip pitch input activity. Higher-frequency Coleman transformations could be easily used within the exact same technique (van Engelen, 2006) to obtain a higher-harmonic controller. In fact, given the reduced inertia of an active tip device, a wider bandwidth control activity could be more easily achieved than using full-span pitch control, especially for very heavy and large blades.

However, a fatigue analysis performed on the reference wind turbine considered in the present study revealed that fatigue is primarily generated in a very low range of frequencies. In fact, Fig. 6 reports the normalized blade root lifetime bending moment damage-equivalent load (DEL) as a function of load harmonics for the baseline RWT. It appears that DEL increases very rapidly with frequency, to the point that $75 \%$ of damage is already accumulated for frequencies up to 1P. Damage then rapidly levels off, with very little contributions coming from frequencies above the 3P. For this reason, and given the preliminary nature of the present study, it was decided to limit the tip control activity here to the sole $1 \mathrm{P}$ harmonic.

\subsection{Tuning of the active tip control law}

Tuning of the cyclic tip pitch controller involves setting the reference values for the direct and quadrature loads, as well as the proportional and integral gains.

Figure 7 shows the $M_{\mathrm{d}}$ (at top) and $M_{\mathrm{q}}$ (at bottom) values vs. wind speed for the baseline wind turbine without tips, using dash-dotted lines. The same figure also shows the reference values $M_{\mathrm{d}}^{*}$ and $M_{\mathrm{q}}^{*}$, using dashed lines. These values were chosen by trial and error and, as previously explained, aim at lowering the feedback loads due to the reduced authority of a tip compared to a full-span pitch control solution.
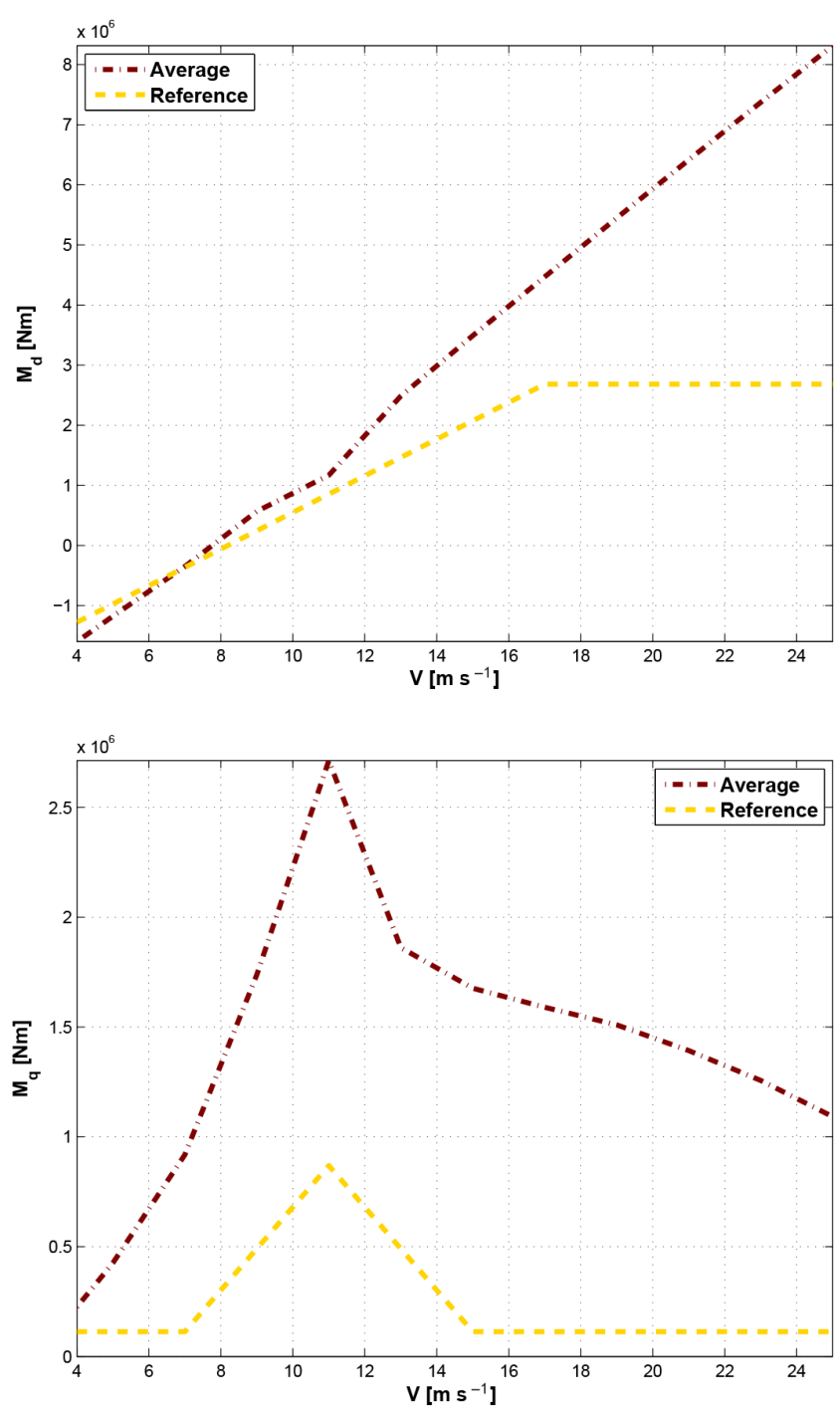

Figure 7. Top: average $M_{\mathrm{d}}$ load (dashed line) and reference value $M_{\mathrm{d}}^{*}$ (dash-dotted line) vs. wind speed. Bottom: average $M_{\mathrm{q}}$ load (dashed line) and reference value $M_{\mathrm{q}}^{*}$ (dash-dotted line) vs. wind speed.

The tip controller was tuned using turbulent wind conditions (DLC (design load case) 1.1, IEC, 2005; GL, 2010). Gains were set in order to achieve satisfactory performance on hub loads, while at the same time avoiding an excessive actuator duty cycle (ADC). The performance of the controller was checked with respect to the most demanding conditions (DLC 1.3). A simple gain scheduling was used to further boost performance, by multiplying the gains by a factor of 4 around rated wind speed, and specifically between 9 and $11 \mathrm{~m} \mathrm{~s}^{-1}$. Due to the lower loads sustained by tip actuators compared to blade root ones, tip IPC was used over the whole operating range of the machine and not only in region III as customarily done for full-span blade IPC. 


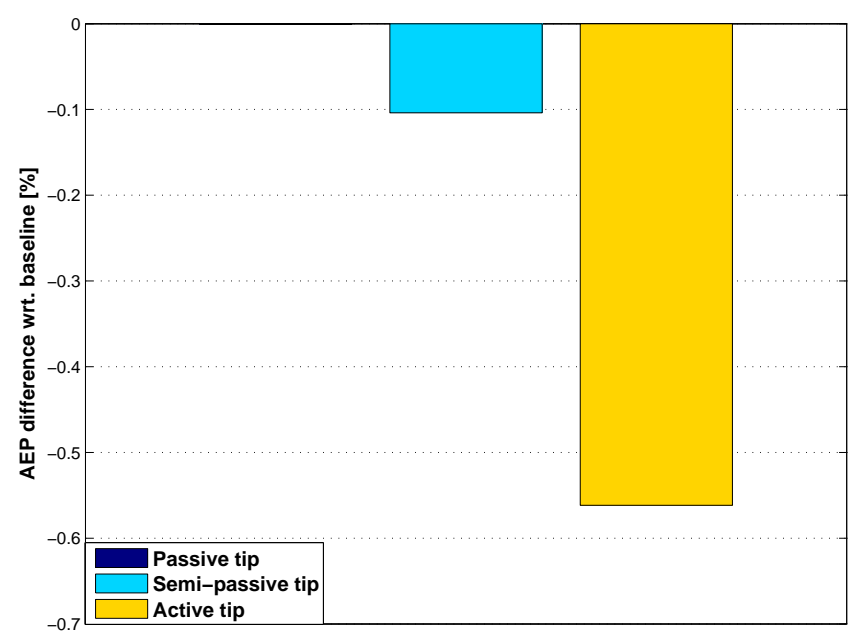

Figure 8. Percent AEP variation with respect to baseline configuration.

Both the reference loads and gains were scheduled using a 30 s moving-averaged wind speed measured from the nacelle anemometer. Fixed-frame loads were low-pass filtered with a fourth-order Butterworth filter with a cut-off frequency of $0.1 \mathrm{~Hz}$

\section{Results}

The performance of the proposed tip devices was evaluated by studying the wind turbine in different operating conditions, as recommended by international certification standards (IEC, 2005). Of all various DLCs used to design the machine (Bak et al., 2013), the most demanding ones in terms of fatigue and ultimate loads were selected. In turbulent wind conditions, results were averaged over four different realizations corresponding to different seeds (Jonkman and Buhl Jr., 2006).

\subsection{Standard design conditions}

The standard power production range was simulated by DLC 1.1 from the cut-in to the cut-out speeds in $2 \mathrm{~m} \mathrm{~s}^{-1}$ increments, without the extrapolation of loads.

The AEP percent variations with respect to the baseline configuration without tip devices are reported in Fig. 8. Apparently, the active tip device has the largest impact on energy capture, possibly due to the choice of operating it also in region II. The maximum AEP reduction is equal to $0.5 \%$, a value that is not insignificant and could offset the advantages in terms of load alleviation. A complete redesign study should be used to combine the variation of AEP with load reductions into a single $\mathrm{CoE}$ value. Note, however, that for the passive and semi-passive solutions AEP reductions are very small and possibly within the margin of accuracy between any two of these simulation analyses.
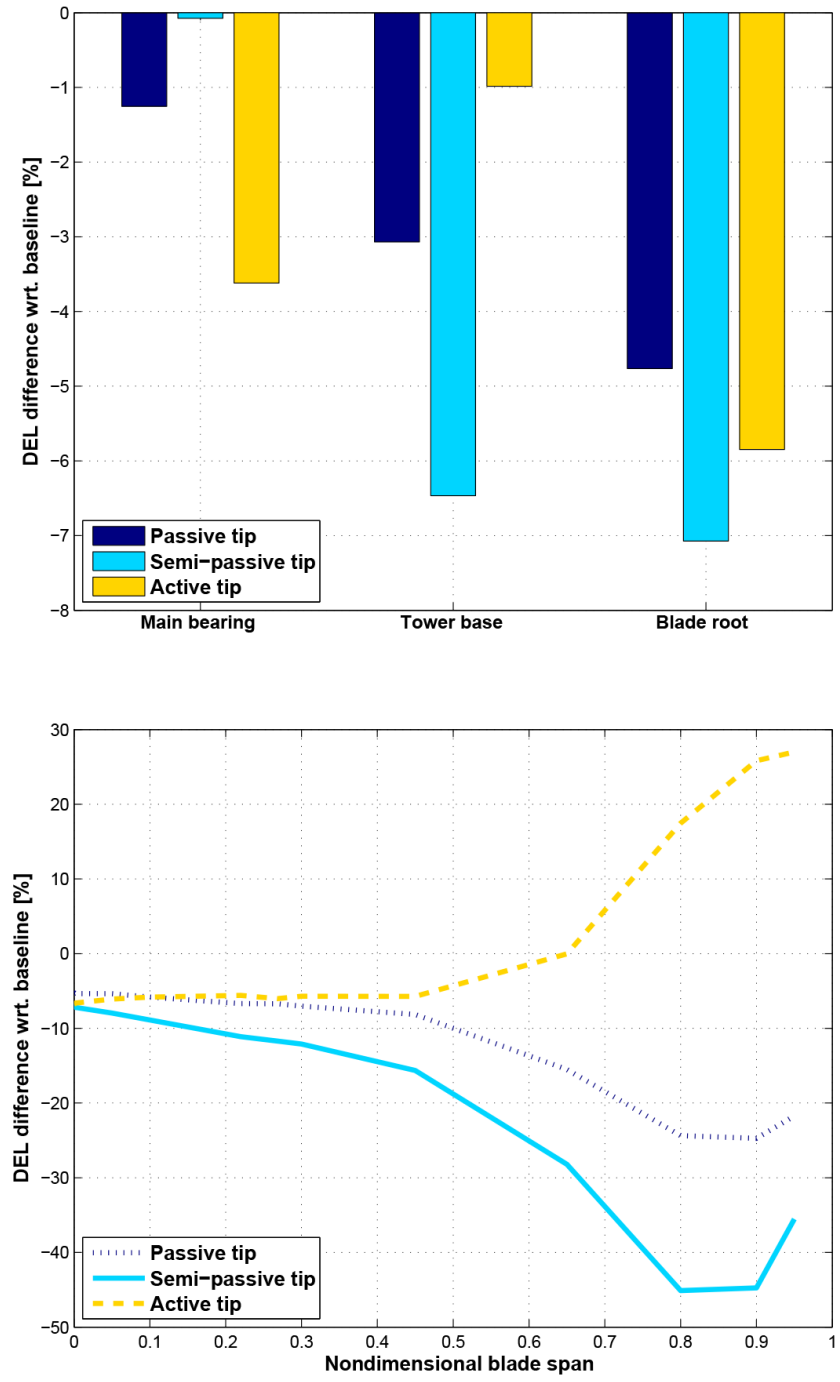

Figure 9. Top: percent variation of DELs at verification spots. Bottom: percent variation of DELs vs. blade span.

DELs were evaluated at a number of points on the machine based on rainflow counting. The blade, main bearing and tower base were selected as fatigue verification spots because they are indicative of possible structural regions prone to fatigue problems. DELs corresponding to the combined moment at the most damaged point at each verification section are reported in Fig. 9.

The effects of the appended devices at the blade root, main bearing and tower base are shown in the top part of the figure. All three tip devices appear to be lowering fatigue loads, although to a different extent at different verification spots. The active tip achieves the best load reduction at the main bearing because those are indeed the loads targeted by the tip IPC control algorithm. On the other hand, it is interesting to observe that the passive and semi-passive tips perform better than the active configuration at the tower base, where the DEL is mainly due to rotor thrust. In fact, these results seem 
to indicate the ability of the passive and semi-passive tips to smooth out load fluctuations due to turbulence. As the three tips operate independently, in contrast with the centralized operation of the IPC algorithm, they are better able to react locally to local wind fluctuations, in turn resulting in smaller fatigue damage at the tower base. The effects at the blade root are also significant, the semi-passive achieving the best results, followed by the active tip and finally closely followed by the fully passive configuration.

However, a more detailed analysis of blade fatigue reveals significant differences among the three solutions, as shown in the bottom part of Fig. 9. In particular, the plot of DEL vs. blade span shows that the passive and semi-passive solutions reduce fatigue throughout the whole span of the blade, which again indicates the ability of the tips to smooth out aerodynamic loads. By contrast, the active tip lowers fatigue towards the root, but increases it at the tip. This is due to the commanded pitch activity that, with the final goal of lowering nodding and yawing moments at the main bearing, in reality overloads the blade tip. Usually fatigue may become a design driver in the inner portion of the blade, so the increase in DEL towards the tip might not be a major source of concern. Nevertheless, a rise in fatigue damage in the tip region should be expected during blade design and would have to be considered.

Pitch activity is reported in Fig. 10 in terms of ADC vs. hub-height wind speed, where ADC over a time span $T$ is computed as

$$
\mathrm{ADC}=\frac{1}{T} \int_{0}^{T}|\dot{\beta}| \mathrm{d} t .
$$

The collective pitch ADC, which measures the blade pitch activity performed by the controller governing the machine (Hansen and Henriksen, 2013), is shown in the top part of the picture. Differences are modest, with some reduction noticeable for the semi-passive solution. This can be attributed once again to the smoothing of the airloads performed by this device, which in turn yields a smoother response of the machine and a consequent slightly reduced activity of the controller in reaction to wind fluctuations.

The bottom part of Fig. 10 shows the tip ADC vs. wind speed. For the passive and semi-passive solutions, ADC is only a measure of how much the tip pitches in response to load fluctuations, while for the active case it represents a measure of the control effort exerted by the actuator. The plot shows that the three devices have very roughly similar tip activities, although these are in nature quite different, as shown by the previous load analysis. In addition, it appears that the semi-passive device has a more pronounced activity than the passive one.

An ultimate load analysis was performed by considering a selected set of DLCs. DLC 1.1 and 1.3 consider power production in standard and extreme turbulence conditions. In
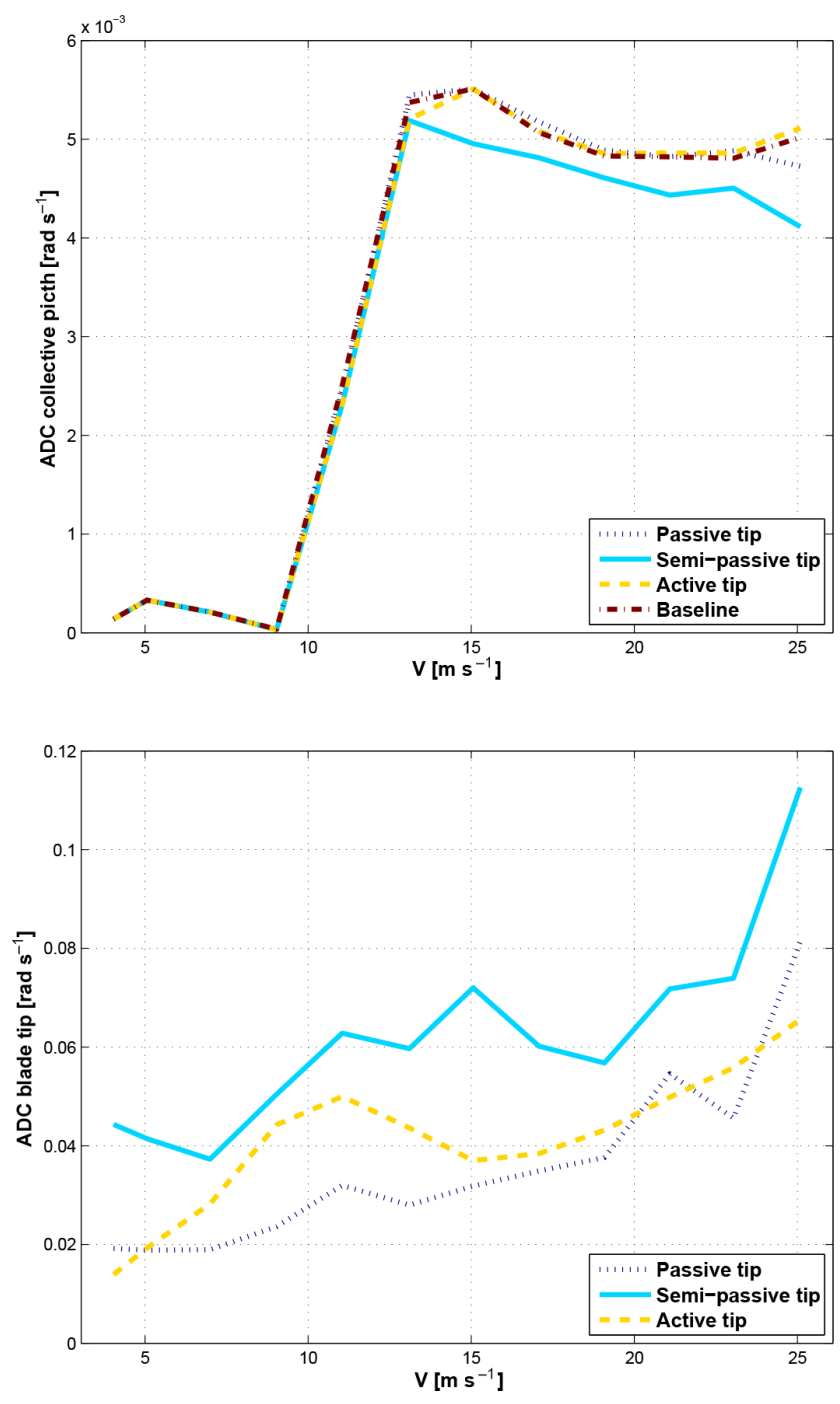

Figure 10. Top: blade pitch ADC vs. wind speed. Bottom: tip ADC vs. wind speed.

DLC 2.3, a deterministic gust occurs in conjunction with a grid loss, and the effects of the fault time are examined by multiple simulations. Finally, DLC 6.2 considers parked conditions with grid failure, where multiple yaw conditions are considered to identify the worst scenario.

Attention is focused on the combined bending moments at the blade root, main bearing and tower base, and percent variations of the ultimate loads with respect to the baseline are reported in Fig. 11. Better performance is achieved at the main bearing and at the blade root, where the most demanding situations are due to DLC 1.3. Here again, as in the case of fatigue damage, the tip devices seem to be able to smooth out airloads, with a beneficial effects also on peak loads.

The situation is different for ultimate loads at the tower base, which are due to DLC 6.2. Although in this case tip oscillations do not in general help in reducing loads, the abil- 


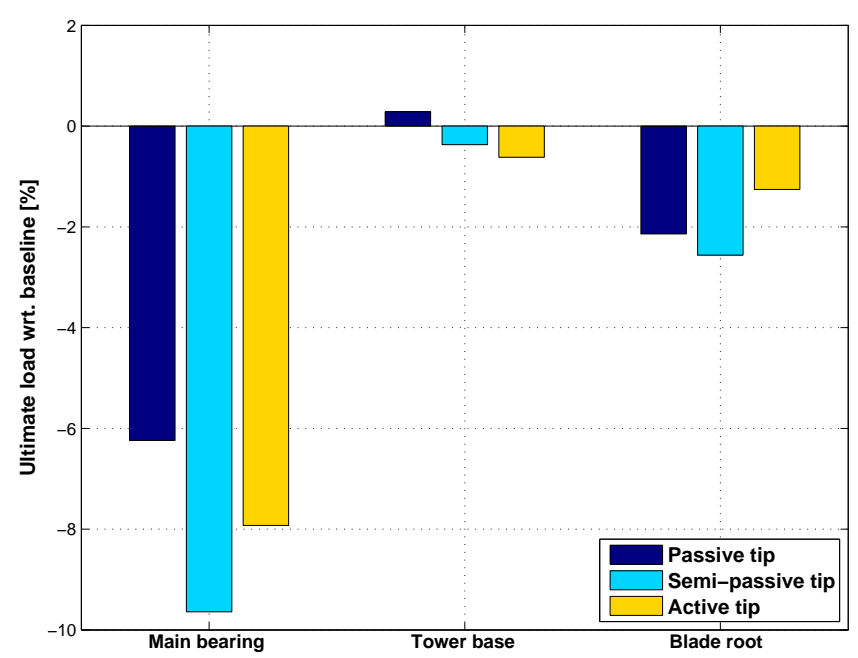

Figure 11. Percent variation of ultimate loads at verification spots with respect to baseline.

ity of the active and semi-passive solutions to deflect the tip can be used to gain a modest advantage. In fact, by pitching the tip one may reduce the sail area of the blade, which in turn may somewhat reduce loads during storms. For these two cases, tips were pitched all the way to their stop positions $\left(20^{\circ}\right)$. As shown by the figure, this strategy results in a modest decrease of loads at the tower base. This active protection of the rotor in storm conditions is not possible with the fully passive solution, where the tip is free to float into the wind but cannot be controlled directly. The same figure shows that this has a very modest negative effect on tower loads.

\subsection{Off-design conditions}

The effects of a blade tip failure are investigated to understand if the advantages of the proposed tip devices can be offset by a fault of the tip pitching system. The fault is investigated by blocking the relative rotation of a single blade tip, while the other ones are functioning in a regular way. It is supposed that the wind turbine is equipped with a safety system to detect the fault and trigger an immediate shut down procedure. Generator fault or loss of electrical network are not included in the fault scenario because simultaneous malfunctions are considered as very unlikely.

Blade tip faults are examined using DLC 2.1 and 2.3 to identify the most critical condition. A single seed is considered for DLC 2.1 normal turbulence model (NTM) simulations because the relative position of the fault with respect to wind fluctuations is more important than the analysis of different wind realizations. The blade tip fault is imposed in conjunction with a positive steep gradient or a maximum of the hub-height wind speed. These two conditions are respectively labeled "grad" and "peak" in the following. When turbulent winds are considered, each simulation is associated with a number, which represents the mean hub-height wind

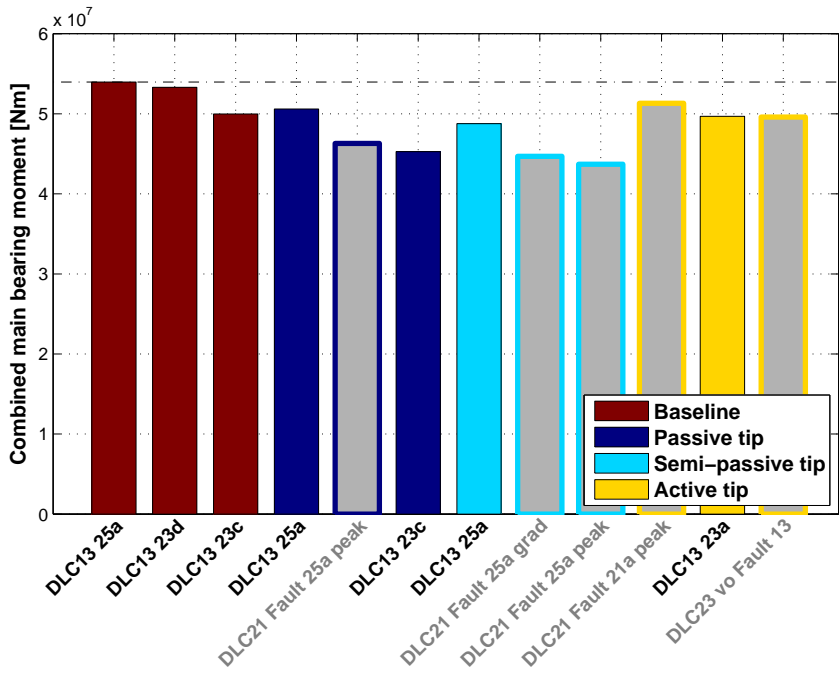

Figure 12. Ranking analysis of main bearing combined moment. Blade tip fault conditions are displayed using gray-shaded bars.

speed, and a letter, identifying a turbulent seed. DLC 2.3 simulates a deterministic extreme operating gust (EOG) at cutout (labeled vo), rated (labeled vr) and rated $\pm 2 \mathrm{~m} \mathrm{~s}^{-1}$ (labeled $\mathrm{vr} \pm 2$ ) wind speed. In total, 16 simulations were performed at each wind speed, varying the time interval between the gust and the fault as well as the azimuthal position of the faulty blade tip. Each simulation is identified by a number that refers to one of these combinations.

The off-design performance is investigated by ranking the ultimate loads of the standard envelope plus the fault conditions in decreasing order and monitoring the variation of the maximum load magnitude. The first three ranking combined moments at the main bearing are reported for each configuration in Fig. 12, where the fault conditions are identified by using gray-shaded bars.

A blade tip failure is considered dangerous if the maximum load magnitude increases with respect to the baseline configuration. The ranking analysis for blades and tower base are not reported here because fault conditions do not modify the five highest-ranking loads. In fact, DLC 1.3 remains the load case driving blade design, while the tower is still stressed by DLC 6.2. By contrast, the combined moment at the main bearing is affected by the rotor imbalance caused by the blade tip fault. Therefore, off-design conditions may generate loads that are comparable to, or even higher than, in the non-faulty standard DLCs.

The results reported in the figure show that all tip devices do not exceed the load envelope of the baseline machine. In addition, fault conditions are not load drivers for the passive and semi-passive solutions, while they produce the leading load for the active tip case. This might be due to the loss of coordination of the blade tip movement that follows a tip fault. 


\section{Conclusions and future work}

A movable blade tip concept for load mitigation on wind turbines has been investigated in this paper. Although already studied for rotorcraft applications, the installation on multiMW HAWTs is, to the authors' knowledge, a novelty. The device allows for a relative pitching motion of the blade tip with respect to the rest of the blade, introducing a further control capability.

Passive, semi-passive and active blade tip solutions were developed and compared. The passive solution achieves the simplest configuration because it does not involve any active component. The active tip requires sensors and servomotors and implements a feedback control algorithm. The semipassive tip is in a sense in between the other two configurations, requiring active slow regulation of the hinge preload but no feedback control. The free motion of the passive and semi-passive devices is driven by the weathercock tendency of the tip due to a suitable chordwise location of its hinge, together with a restraining spring. These devices result in a passive decentralized control strategy powered by local fluctuation of the aerodynamic loads. The resulting tip pitching smooths out the airloads without incurring in AEP losses of any significance. By contrast, the active tip implements a centralized IPC control strategy, that targets the nodding and yawing moments at the hub and seems associated with a more significant impact on AEP.

The paper described the preliminary sizing of all devices. The hinge preload and stiffness for the passive and semipassive configurations were defined by an ad hoc procedure, while simple guidelines were reported for the tuning of the gains of the active tip system.

The devices were tested in a comprehensive simulation environment, with application to a large conceptual future machine. The analysis considered both fatigue and ultimate loads, including also tip fault conditions, following accepted standard certification guidelines.

Based on the results of the present analysis, the following conclusions may be drawn:

- All proposed tip devices improve on the baseline both in terms of fatigue and ultimate load alleviation, although to a different extent on different wind turbine components. These results might possibly be further improved by a more complete optimization of the devices, including their aerodynamic shape.

- The more significant effects on fatigue are reported at the blade root and tower base. For the passive and semi-passive devices, this seems to be attributable to a smoothing of the airloads. Ultimate loads see the largest decrease at the main bearing, while they are essentially unaffected on blade and tower.

- None of the devices seems to significantly interfere with the collective pitch-torque control system used for reg- ulating the machine, although no retuning of the controller was performed. For the semi-passive solution, the load smoothing generated by the tip results in a slightly reduced duty cycle of the blade pitch actuator.

- The consequences of a blade tip fault are limited, with no effect on the ultimate design-driving loads. The active and semi-active devices can be used to reduce blade sail area in storm conditions. Although this technique did not reduce ultimate loads on this specific machine, it might be beneficial to other wind turbines more significantly driven by storm conditions.

Further studies are clearly necessary before final conclusions may be drawn, although these initial results seem to be promising. In particular, the passive and semi-passive solutions behave nearly as well as the active one, at a reduced complexity. This might be interesting for applications where reliability, low cost of maintenance and high availability are important, as in the offshore case.

The blade tip concept could be further developed along different lines. The detailed design of the tip joint should be performed, addressing some critical aspects such as the realization of the passive screw joint or the installation of the servomotors. More sophisticated aerodynamic models could be used to take into account the mutual interference between the tip and the inner part of the blade, as well as the vortices shed by the twist discontinuity at the joint (van Aken and Stroub, 1986). The control system could be retuned to better account for the presence of the tip devices, while shutdown procedures could also be revisited at least in the active tip case. Finally, the integration of the blade tip concept in a rotor redesign activity (Bottasso et al., 2015a) could shed light on the actual potential beneficial effects on $\mathrm{CoE}$ or the lack thereof.

\section{Nomenclature}

$\begin{array}{ll}F & \text { Force } \\ g & \text { Gravitational acceleration } \\ J & \text { Moment of inertia } \\ k & \text { Control gain } \\ K & \text { Stiffness } \\ m & \text { Mass } \\ M & \text { Moment } \\ r & \text { Radial position } \\ T & \text { Time interval } \\ V & \text { Wind speed } \\ z & \text { Tip spanwise displacement } \\ \beta & \text { Blade pitch angle } \\ \theta & \text { Blade tip relative rotation } \\ \psi & \text { Blade azimuth } \\ \tau & \text { Transmission ratio } \\ \omega & \text { Modal frequency }\end{array}$


$\Omega \quad$ Rotor angular speed

(.) Derivative with respect to time, $\mathrm{d} \cdot / \mathrm{d} t$

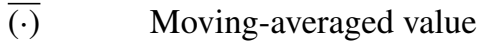

$(\cdot)^{*} \quad$ Reference value

$(\cdot)_{\mathrm{a}} \quad$ Aerodynamic term

$(\cdot)_{\mathrm{c}} \quad$ Centrifugal term

$(\cdot)_{\mathrm{d}} \quad$ Direct (yawing) term

$(\cdot)_{\mathrm{g}} \quad$ Gravity term

$(\cdot)_{\mathrm{q}} \quad$ Quadrature (nodding) term

$(\cdot)_{\mathrm{PT}} \quad$ Passive tip term

$(\cdot)_{\mathrm{SP}} \quad$ Semi-passive tip term

AC Aerodynamic center

ADC Actuator duty cycle

AEP Annual energy production

BEM Blade element momentum

BTC Bend-twist coupling

CG Center of gravity

CoE Cost of energy

DEL Damage-equivalent load

DLC Dynamic load case

EOG Extreme operating gust

HAWT Horizontal axis wind turbine

HL Hinge line

IPC Individual pitch control

NTM Normal turbulence model

NWP Normal wind profile

RWT Reference wind turbine

\section{Data availability}

Data will be made available upon request.

Acknowledgement. The present work was partially supported at the Politecnico di Milano by the FP7 INNWIND.EU project.

Edited by: G. J. W. van Bussel

Reviewed by: two anonymous referees

\section{References}

Andersen, P. B., Henriksen, L., Gaunaa, M., Bak, C., and Buhl, T.: Deformable trailing edge flaps for modern megawatt wind turbine controllers using strain gauge sensors, Wind Energy, 13, 193-206, doi:10.1002/we.371, 2010.

Arrieta, A. F., Kuder, I. K., Rist, M., Waeber, T., and Ermanni, P.: Passive load alleviation aerofoil concept with variable stiffness multi-stable composites, Compos. Struct., 116, 235-242, doi:10.1016/j.compstruct.2014.05.016, 2014.

Bak, C., Zahle, F., Bitsche, R., Kim, T., Yde, A., Henriksen, L. C., Andersen, P. B., Natarajan, A., and Hansen, M. H.: Description of the DTU 10MW Reference Wind Turbine, Danish Wind Power Research 2013, Fredericia, Denmark, DTU Wind Energy Report-I-0092, 2013.

Barlas, T. K., van der Veen, G. J., and van Kuik, G. A. M.: Model predictive control for wind turbines with distributed active flaps: incorporating inflow signals and actuator constraints, Wind Energy, 15, 757-771, doi:10.1002/we.503, 2011.

Bergami, L. and Poulsen, N. K.: A smart rotor configuration with linear quadratic control of adaptive trailing edge flaps for active load alleviation, Wind Energy, 18, 625-641, doi:10.1002/we.1716, 2015.

Bielawa, R. L.: Analytic Investigation of Helicopter Appended Aeroelastic Devices, NASA Ames Research Center, NASA CR166525, 96 pp., 1984.

Bossanyi, E.: Wind turbine control for load reduction, Wind Energy, 6, 229-244, doi:10.1002/we.95, 2003a.

Bossanyi, E.: Individual blade pitch control for load reduction, Wind Energy, 6, 119-128, doi:10.1002/we.76, 2003 b.

Bossanyi, E.: Further load reductions with individual pitch control, Wind Energy, 8, 481-485, doi:10.1002/we.166, 2005.

Bottasso, C. L., Croce, A., Savini, B., Sirchi, W., and Trainelli, L.: Aero-servo-elastic modeling and control of wind turbines using finite-element multibody procedures, Multibody Syst. Dyn., 16, 291-308, doi:10.1007/s11044-006-9027-1, 2006.

Bottasso, C. L., Croce, A., Nam, Y., and Riboldi, C. E. D.: Power curve tracking in the presence of a tip speed constraint, Renew. Energ., 40, 1-12, doi:10.1016/j.renene.2011.07.045, 2011.

Bottasso, C. L., Campagnolo, F., Croce, A., and Tibaldi, C.: Optimization-based study of bend-twist coupled rotor blades for passive and integrated passive/active load alleviation, Wind Energy, 16, 1149-1166, doi:10.1002/we.1543, 2013a.

Bottasso, C. L., Croce, A., Riboldi, C. E. D., and Nam, Y.: Multilayer control architecture for the reduction of deterministic and non-deterministic loads on wind turbines, Renew. Energ., 51, 159-169, doi:10.1016/j.renene.2012.08.079, 2013 b.

Bottasso, C. L., Bortolotti, P., Croce, A., and Gualdoni, F.: Integrated aero-structural optimization of wind turbines, Multibody Syst. Dyn., 1-28, doi:10.1007/s11044-015-9488-1, 2015a.

Bottasso, C. L., Croce, A., Gualdoni, F., and Montinari, P.: Load mitigation for wind turbines by a passive aeroelastic device, J. Wind Eng. Ind. Aerod., 148, 57-69, doi:10.1016/j.jweia.2015.11.001, 2015b.

Chow, R. and van Dam, C. P.: Computational investigations of deploying load control microtabs on a wind turbine airfoil, 45th AIAA Aerospace Sciences Meeting and Exhibit, Reno, NV, doi:10.2514/6.2007-1018, 2007.

Donely, P. and Shufflebarger, C. C.: Test of a Gust-Alleviating Flap in the Gust Tunnel, NACA, Washington, D.C., Technical Note No. 745, 24 pp., 1940.

DTU RWT: DTU 10 MW Reference Wind Turbine Project, available at: http://dtu-10mw-rwt.vindenergi.dtu.dk, last access: 18 November 2016, 2015.

GL (Germanischer Lloyd): Guideline for the Certification of Wind Turbines, Edition 2010, Germanischer Lloyd Industrial Services GmbH, Renewables Certification, Brooktorkai 10, 20457 Hamburg, Germany, 2010.

Hansen, M. O. L.: Aerodynamics of Wind Turbines, 2nd Edn., Earthscan, London, UK, and Sterling, VA, 2008.

Hansen, M. H. and Henriksen, L. C.: Basic DTU Wind Energy controller, DTU Wind Energy Report-E-0018, DTU Wind Energy, 2013.

Hagg, F., Joosse, P. A., van Kuik, G. A. M., Beurskens, J. H. M., and Dekker, J. W. M.: The results of the Dutch exhat program, the 
technology for the next generation of wind turbines, Proceedings of Windpower'93, San Francisco, CA, 1993.

IEC (International Electrotechnical Commission): IEC 61400-1: Wind Turbines - Part 1: Design Requirements, 3rd Edn., International Standard, IEC 61400-1, 2005.

Johnson, W.: Rotorcraft Aeromechanics, Cambridge University Press, New York, NY, USA, 2013.

Jonkman, B. J. and Buhl Jr., M. L.: TurbSim user's guide, National Renewable Energy Laboratory, Golden, CO, Technical Report NREL/TP-500-39797, 2006.

Konstanzer, P., Enenkl, B., Aubourg, P. A., and Cranga, P.: Recent advances in Eurocopter's passive and active vibration control, American Helicopter Society 64th Annual Forum, Montréal, Canada, 29 April-1 May 2008.

Lachenal, X., Daynes, S., and Weaver, P. M.: Review of morphing concepts and materials for wind turbine blade applications, Wind Energy, 16, 283-307, doi:10.1002/we.531, 2013.

Lackner, M. A. and van Kuik, G.: A comparison of smart rotor control approaches using trailing edge flaps and individual pitch control, Wind Energy, 13, 117-134, doi:10.1002/we.353, 2010.

Lambie, B., Jain, P., and Tropea, C.: Passive camber change for wind turbine load alleviation, 49th AIAA Aerospace Sciences Meeting including the New Horizons Forum and Aerospace Exposition, Orlando, Florida, doi:10.2514/6.2011-637, 2011.

Leithead, W. E., Neilson, V., and Dominguez, S.: Alleviation of unbalanced rotor loads by single blade controllers. European Wind Energy Conference (EWEC 2009), Marseille, France, 2009.

Louie, A.: An Experimental and Analytical Evaluation of the Tapered Tension-Torsion Strap Concept, NASA TM-101049, 18 pp., 1988.

Marten, D., Spiegelberg, H., Pechlivanoglou, G., Nayeri, C. N., Paschereit, C. O., and Tropea, C.: Configuration and Numerical Investigation of the Adaptive Camber Airfoil as Passive Load Alleviation Mechanism for Wind Turbines, 33rd AIAA Applied Aerodynamics Conference, doi:10.2514/6.2015-3390, 2015.
Martin, D. M. and Fortin, P. E.: VSAERO Analysis of tip Planforms for the Free-Tip Rotor, NASA CR 177487, 127 pp., 1988.

Stroub, R. H.: An Analytical Investigation of the Free-Tip Rotor for Helicopters, NASA TM-81345, 34 pp., 1982.

Stroub, R. H.: Analysis of the Free-Tip Rotor Wind-Tunnel Test Results, NASA TM-86751, 70 pp., 1985.

Stroub, R. H., Kumagai, H., and Keys, C.: Wind tunnel test of a model rotor with a free-tip, 4th Applied Aerodynamics Conference, San Diego, CA, doi:10.2514/6.1986-1781, 1986.

Thresher, R., Schreck, S., Robinson, M., and Veers, P.: Wind energy status and future wind engineering challenges, 1st American Association for Wind Engineering Workshop, 21-22 August, Vail, CO, Conference Paper NREL/CP-500-43799, 2008.

Young, L. A.: The Evaluation of a Number of Prototypes for the Free-Tip Rotor Constant-Moment Controller, NASA TM-86664, 76 pp., 1986.

van Aken, J. M. and Stroub, R. H.: Tip Aerodynamics From Wind Tunnel Test of Semi-Span Wing, NASA TM-88253, 20 pp., 1986.

van Engelen, T. G.: Design model and load reduction assessment for multi-rotational mode individual pitch control (higher harmonics control). Proceeding of European Wind Energy Conference (EWEC 2006), Athens, Greece, 27 February-2 March 2006.

van Kuik, G. A. M. and Dekker, J. W. M.: The FLEXHAT program, technology development and testing of flexible rotor systems with fast passive pitch control, J. Wind Eng. Ind. Aerod., 39, 435-448, doi:10.1016/0167-6105(92)90567-T, 1992. 